\title{
Factores que afectan el aprendizaje del idioma inglés en colegios públicos de Quito
}

\section{Factores que afectan el aprendizaje del idioma inglés en colegios públicos de Quito} Verónica Gabriela Páez Romero. ${ }^{1}$ \& Washington Edy Santillán Marroquín. ${ }^{2}$

\begin{abstract}
.
At some time, all we have considered this one question, because we would have to study the English language in our Latin cultures? without a doubt that we will have some interesting answers on the matter like for example: in order to obtain new and better labor options, we will have access to multinational companies.

Really, in the labor thing, to know English, always will have a competitive advantage forehead to other candidates. On the other hand, to speak English is to speak a universal language that opens to an endless number of doors and possibilities to you in the world and all the scopes of the life, in areas like: commercial, financial, political, education, culture, tourism, human investigation, relations, etc. He is rewarding to learn and to dominate a new language and, in the case of the English is an accessible language, as long as you have methodologic motivation, time and other resources that allow you to do it. This investigation must like intention analyze the factors that affect the education process learning of the English language in five public secondary schools of Quito. The main obtained findings are, the size of the classrooms of classes and the number of students who conform these classrooms. In the last years, the development of the technologies of the information, the use of diverse resources playful of specialized education-learning, professors, they have allowed to improve and to enrich the learning of this universal language. These resources applied actually and in our contexts of public education will allow us to facilitate in our children and adolescents the educationlearning of the English.
\end{abstract}

Keywords: Education, factors, school's public, process, playful, technology.

\footnotetext{
${ }^{1}$ Instituto Superior Tecnológico Bernardo O’Higgins. Quito, Ecuador. coordinacion.academica@institutoohiggins.com

${ }^{2}$ Instituto Superior Tecnológico Bernardo O'Higgins. Quito, Ecuador. coordinacion.academica@instituto-ohiggins.com
} 


\section{Resumen.}

¿En algún momento, todos nos hemos planteado ésta pregunta, porqué deberíamos estudiar el idioma inglés en nuestras culturas latinas? sin duda que tendremos algunas respuestas interesantes al respecto como, por ejemplo: para obtener nuevas y mejores opciones laborales, tendremos acceso a empresas multinacionales. En definitiva, en lo laboral, saber inglés, siempre tendrá una ventaja competitiva frente a otros candidatos. Por otro lado, hablar inglés es hablar un idioma universal que te abre un sinnúmero de puertas y posibilidades en el mundo y en todos los ámbitos de la vida, en áreas como: comercial, financiera, política, educación, cultura, turismo, investigación, relaciones humanas, etc.

Es gratificante aprender y dominar un nuevo idioma y, en el caso del inglés es un idioma accesible, siempre y cuando tengas motivación, tiempo y otros recursos metodológicos que te permita hacerlo. Esta investigación tiene como propósito analizar los factores que afectan el proceso de enseñanza- aprendizaje del idioma inglés en cinco escuelas secundarias públicas de Quito. Los principales hallazgos obtenidos son, el tamaño de las aulas de clases y el número de alumnos que conforman dichas aulas. En los últimos años, el desarrollo de las tecnologías de la información, el empleo de diversos recursos lúdicos de enseñanza-aprendizaje, profesores especializados, han permitido mejorar y enriquecer el aprendizaje de este idioma universal. Estos recursos aplicados en la práctica y en nuestros contextos de educación pública nos permitirán facilitar en nuestros niños y adolescentes la enseñanza-aprendizaje del inglés.

Palabras claves: Enseñanza, factores, colegios públicos, proceso, lúdicos, tecnología.

\section{Introducción.}

La mayoría de los estudiantes de Ecuador que terminan la escuela secundaria reconocen que no tienen un buen nivel de inglés que les permita tener un desarrollo académico superior efectivo. En consecuencia, los estudiantes a menudo pierden muchas oportunidades, como trabajos con mejores pagos, viajes, programas de inmersión, oportunidades para mejorar su creatividad y confianza en sí mismos para desarrollar cualquier trabajo que involucre inglés. Por esta razón, es esencial realizar una investigación sobre los factores que afectan el proceso de aprendizaje de la enseñanza del idioma inglés, especialmente en las escuelas secundarias públicas de Quito, a fin de descubrir algunas alternativas prácticas que ayuden a mejorar el inglés de los estudiantes. Nivel y al proceso de enseñanza - aprendizaje.

Para llevar a cabo esta investigación, se consideraron algunos objetivos específicos. El primero fue determinar las condiciones en el aula en las que tienen lugar las clases de inglés. El segundo fue identificar las características de los profesores de inglés en servicio. Y el tercero fue determinar la competencia de los docentes.

Investigaciones previas que se han hecho sobre los factores que afectan al proceso de enseñanza-aprendizaje de inglés, indica que hay diferentes tipos de factores que deben tenerse en cuenta para obtener resultados positivos en dicha enseñanza-aprendizaje. Estos factores 
están relacionados con los maestros, los estudiantes y el entorno en el que se desarrolla la clase impartida.

De esta manera, Ames y Archer (1988) revelan en su estudio cómo los patrones de motivación específicos se relacionan con la prominencia del dominio y las metas de desempeño en los entornos reales del aula, lo que proporciona una manera significativa de diferenciar las percepciones de los estudiantes sobre el entorno de aprendizaje en el aula. Estas percepciones mostraron diferentes patrones de relación con las estrategias de aprendizaje, preferencia por tareas desafiantes, actitud hacia la clase y creencias sobre la causa del éxito y el fracaso. No hubo ninguna limitación en el desarrollo de este estudio.

Además, el estudio de Snow (2002) se realizó con el propósito de conocer las percepciones y experiencias de los maestros de secundaria, en relación con el uso del espacio en el aula, y aclarar que los tamaños de clase más pequeños contribuyen al sentido de bienestar de los maestros y la efectividad, mientras que el mantenimiento deficiente y el hacinamiento se asociaron con sentimientos de frustración. El autor no tuvo ninguna limitación en este estudio.

Finalmente, Abella y Salinas (2006) presentaron otro estudio de caso relacionado con los estilos de aprendizaje en estudiantes de bajo nivel en una escuela privada en Bogotá, cuyo objetivo principal fue descubrir qué roles desempeñan dichos estilos en el proceso de aprendizaje del idioma inglés para los estudiantes con bajo rendimiento académico y, qué factores permiten a estos estudiantes explorar sus estilos de estudio durante el proceso de aprendizaje del idioma inglés. Si bien, hubo algunas limitaciones, los escritores concluyen que la incorporación de estilos de aprendizaje facilitó el proceso de aprendizaje.

El proceso de enseñanza-aprendizaje dará estímulo a maestros, instituciones educativas y autoridades que serán responsables de aplicar las estrategias correctas para satisfacer las necesidades de los estudiantes, una capacitación continua de los maestros, una compra de equipos institucionales eficientes y el desarrollo de tácticas que mejorarán el proceso mencionado.

Por esta razón, esta investigación ofrece varios beneficios para profesores, estudiantes y para instituciones educativas; ya que el objetivo es tener una búsqueda constante de una mejora de sus conocimientos de inglés en cada etapa de la educación. Además, los maestros y los estudiantes podrían tener un nivel óptimo con estándares internacionales técnicamente establecidos.

Por otro lado, las instituciones educativas dieron el apoyo necesario a los investigadores durante su investigación de campo, por lo que, afortunadamente, no tuvieron ningún tipo de limitaciones en el proceso.

\section{Metodología.}

Esta investigación se realizó en cinco escuelas secundarias públicas ubicadas en el centro histórico de la ciudad de Quito. Los datos seleccionados para esta investigación fueron quince profesores y estudiantes de inglés de $8^{\circ}, 9^{\circ}$ y $10^{\circ}$ de educación básica, y $1^{\circ}, 2^{\circ}$ y $3^{\circ}$ de secundaria 
superior. Además, los estudiantes tenían un rango de edad entre 12 y 17 años. Esta investigación se encuentra apoyada por material bibliográfico que fue investigado y obtenido en muchas bibliotecas de diferentes universidades, la Embajada de los Estados Unidos y las instituciones educativas públicas. La revisión de este material bibliográfico fue necesaria para construir la revisión de la literatura, que es la base científica para emitir comentarios sobre el tema investigado.

Los principales temas que apoyan esta tesis son: la importancia de enseñar inglés como idioma extranjero en Ecuador, métodos de enseñanza, técnicas de enseñanza, gestión del aprendizaje, diseño de lecciones, tamaño de la clase, espacio en el aula, disposición de los asientos, recursos lúdicos de enseñanza, observación en el aula, motivación de los alumnos, estilos de aprendizaje e inteligencias múltiples de los alumnos.

El enfoque general utilizado en esta investigación fue de campo y se utilizó herramientas cuantitativas. Las técnicas utilizadas para la recopilación de datos fueron encuestas, observación, entrevista y proceso de toma de notas, junto con instrumentos tales como hojas de observación, un cuestionario para estudiantes y otro para maestros, y un formato de entrevista.

En primer lugar, se pidió a quince maestros y quince estudiantes que respondieran un cuestionario. El cuestionario de los profesores consistió en veinte preguntas en inglés sobre los diferentes factores que afectan el proceso de enseñanza-aprendizaje del idioma inglés, y el cuestionario de los estudiantes consistió en catorce preguntas en español sobre los factores que afectan el proceso de enseñanza-aprendizaje del idioma inglés.

Al mismo tiempo, los investigadores tenían sus propias hojas con todos los detalles de las observaciones hechas a los maestros y sus alumnos en el aula. Se aplicó una técnica de observación y concentración meticulosa para tener en cuenta todos los detalles que contenían el proceso de enseñanza-aprendizaje del idioma inglés.

\section{Resultados.}

La tabulación de los datos se basó en las quince clases observadas, y todos los resultados obtenidos se tuvieron en cuenta con el propósito de desarrollar las estadísticas correspondientes y realizar un proceso de mejora continua.

El aspecto considerado para el análisis de los resultados fue la revisión en forma detallada de las respuestas dadas por los profesores y los estudiantes, respaldando esta información con las citas de diferentes autores recopiladas de la revisión de la literatura, las observaciones que los investigadores hicieron en el aula durante la clase impartida, y con la opinión personal de los investigadores según sus percepciones de acuerdo con los temas investigados y todos los conocimientos adquiridos.

\section{Análisis cuantitativo}

\section{Factores concernientes a los maestros}


Figura 1. ¿Qué nivel de educación tienen los profesores?

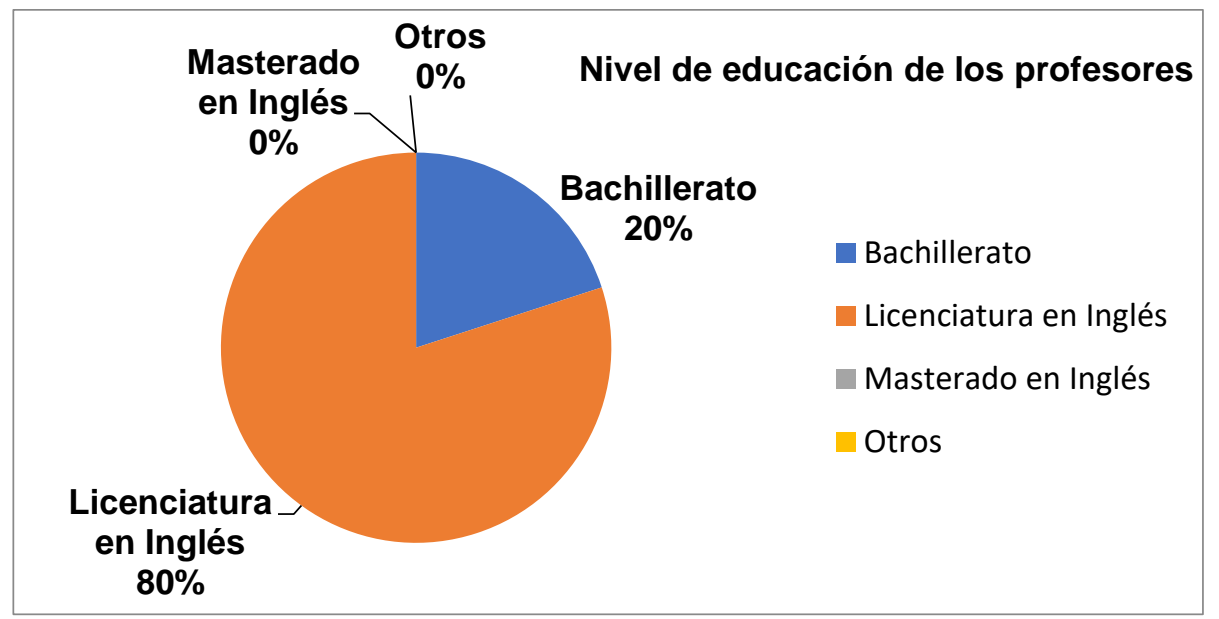

Fuente: Elaboración propia.

Figura 2. ¿Cuál de los siguientes métodos fue usado en las clases observadas?

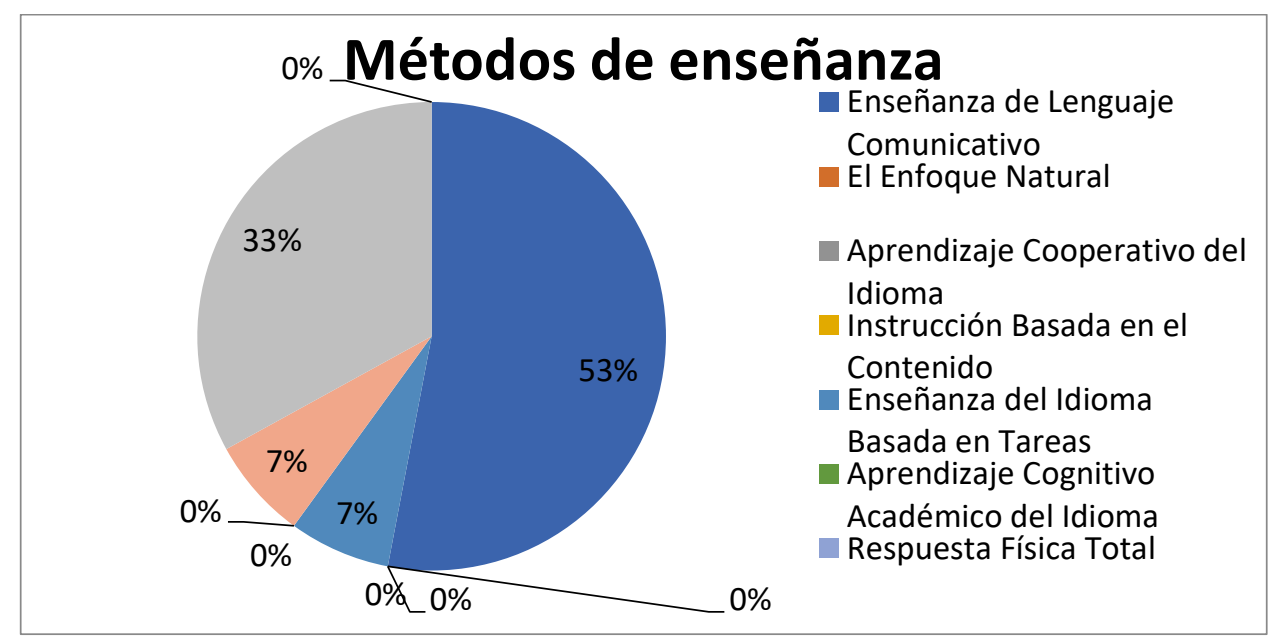

Fuente: Elaboración propia.

Figura 3 ¿Los maestros usan actividades de trabajo en grupo para enseñar sus lecciones?

\section{Actividades en grupo}

Fuente: Elaboración propia. 
Figura 4. ¿Los maestros usan actividades individuales para enseñar sus lecciones?

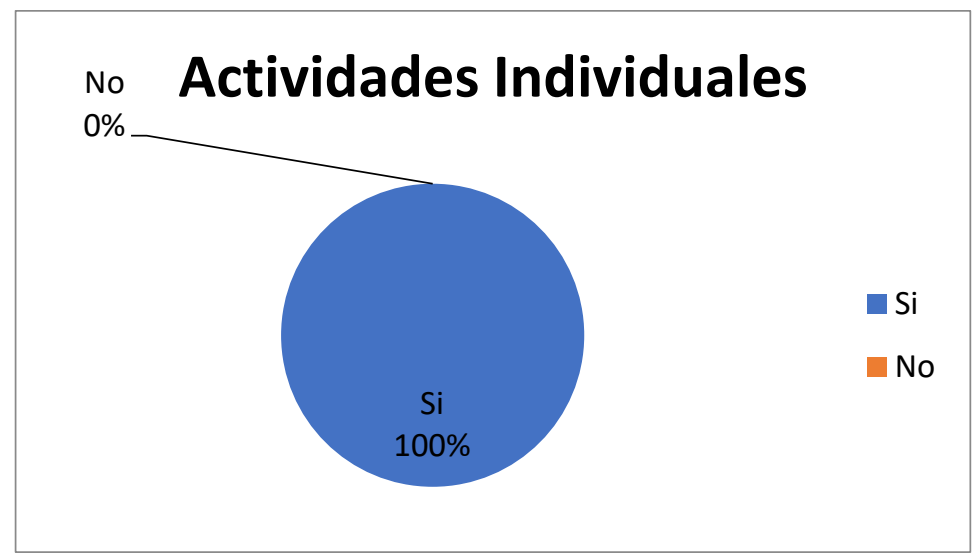

Fuente: Elaboración propia.

Figura 5 ¿Los maestros usan actividades de trabajo en grupo para enseñar sus lecciones?

\section{Trabajo en grupo}

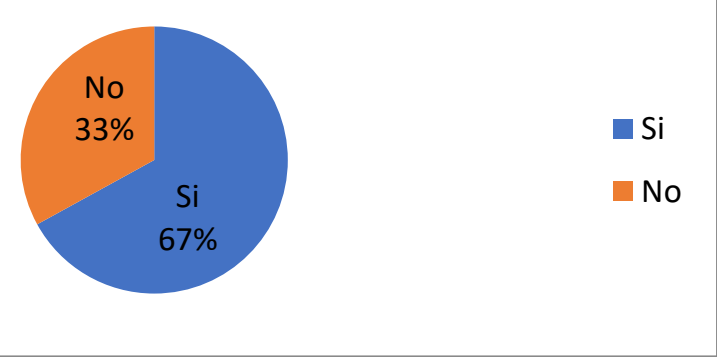

Fuente: Elaboración propia.

Figura 6 ¿Los maestros usan el inglés la mayor parte del tiempo en sus clases?

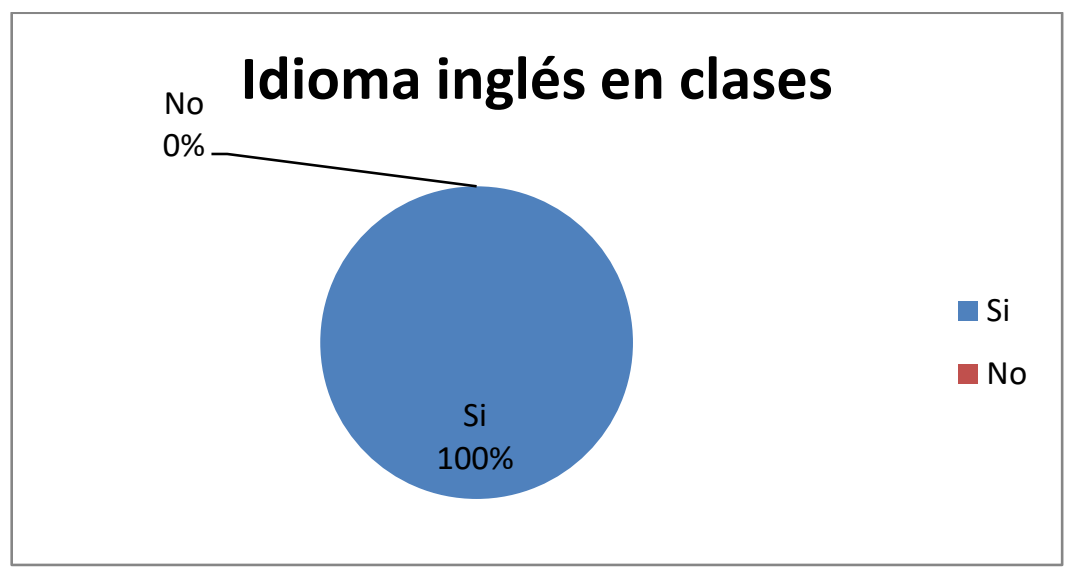

Fuente: Elaboración propia. 
Figura 7 ¿Los maestros planean sus lecciones?

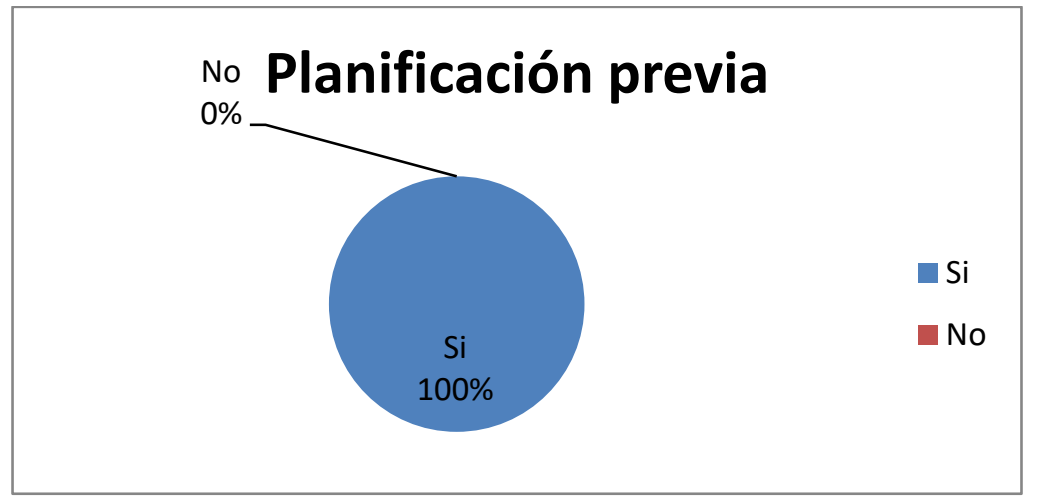

Fuente: Elaboración propia.

Figura 8. ¿Los maestros consideran aspectos tales como disciplina, tiempo, retroalimentación e instrucciones para enseñar sus lecciones?

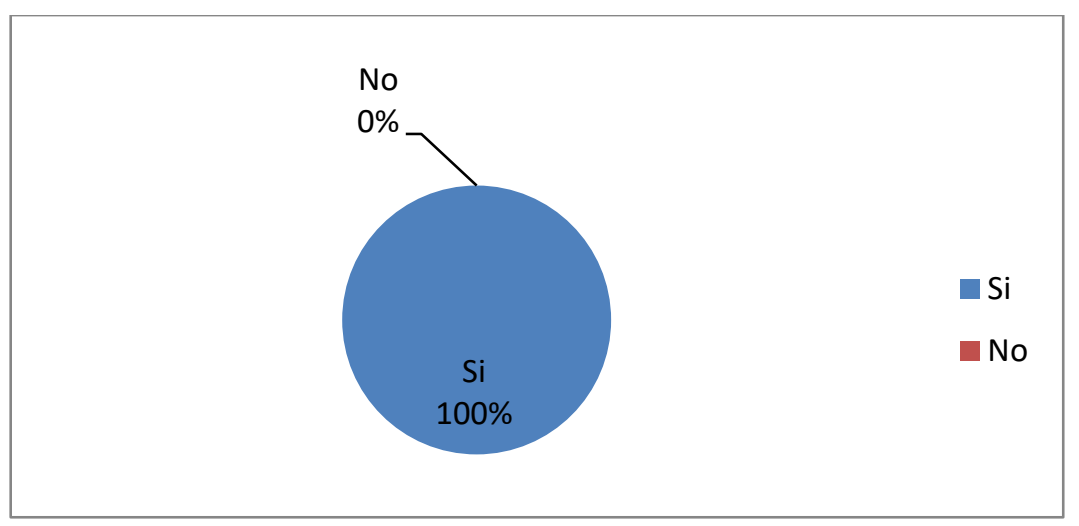

Fuente: Elaboración propia.

Figura 9. ¿Los maestros consideran las necesidades de los estudiantes para enseñar inglés con éxito?

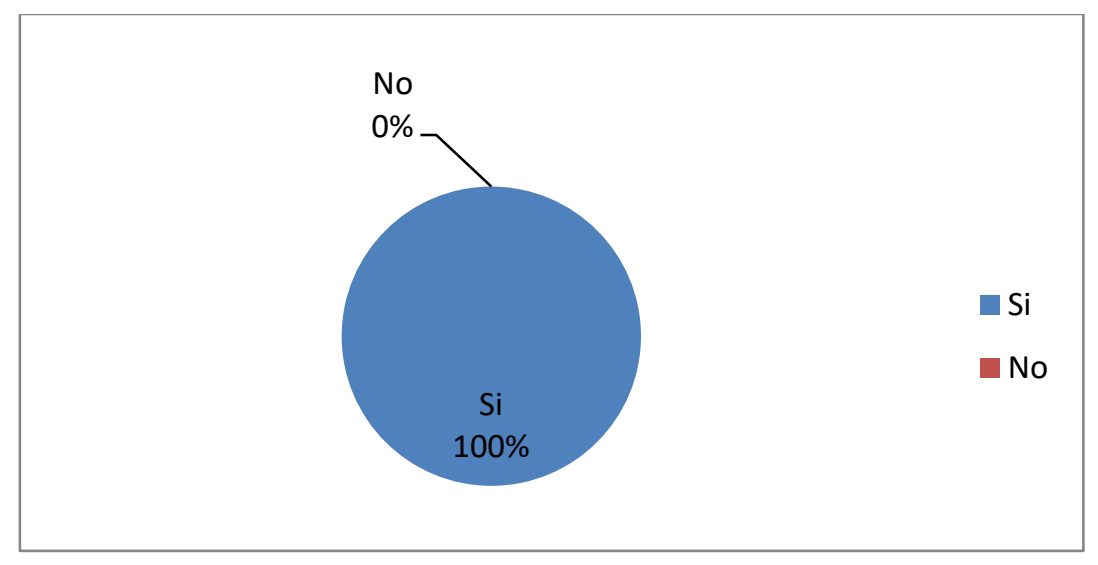

Fuente: Elaboración propia. 
Figura 10. ¿Consideran los maestros el nivel de los estudiantes para enseñar inglés con éxito?

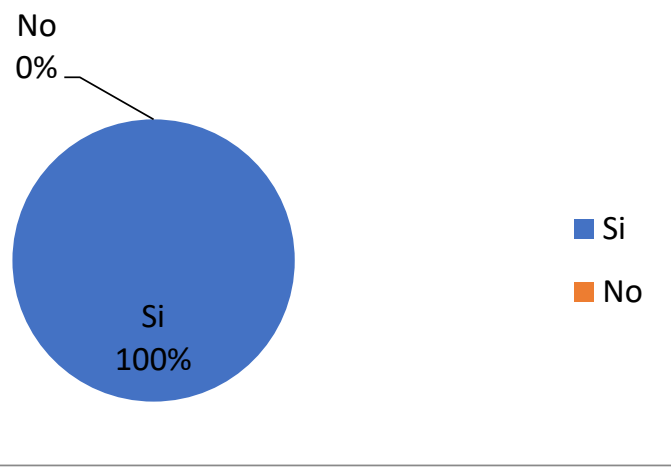

Fuente: Elaboración propia.

Figura 11. ¿Cuál es el nivel de sus alumnos?

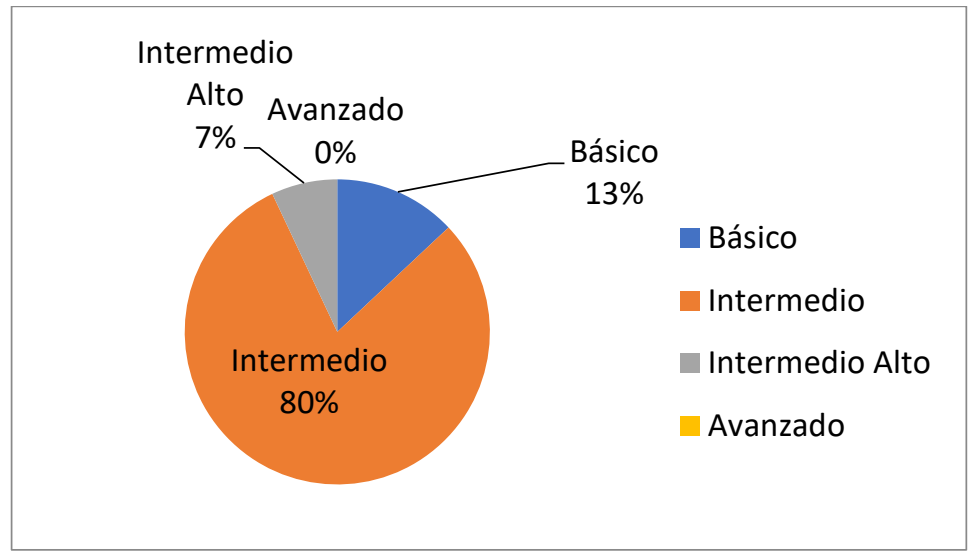

Fuente: Elaboración propia.

\section{Factores concernientes a las aulas}

Figura 12. ¿Cuántos estudiantes hay en las clases observadas?

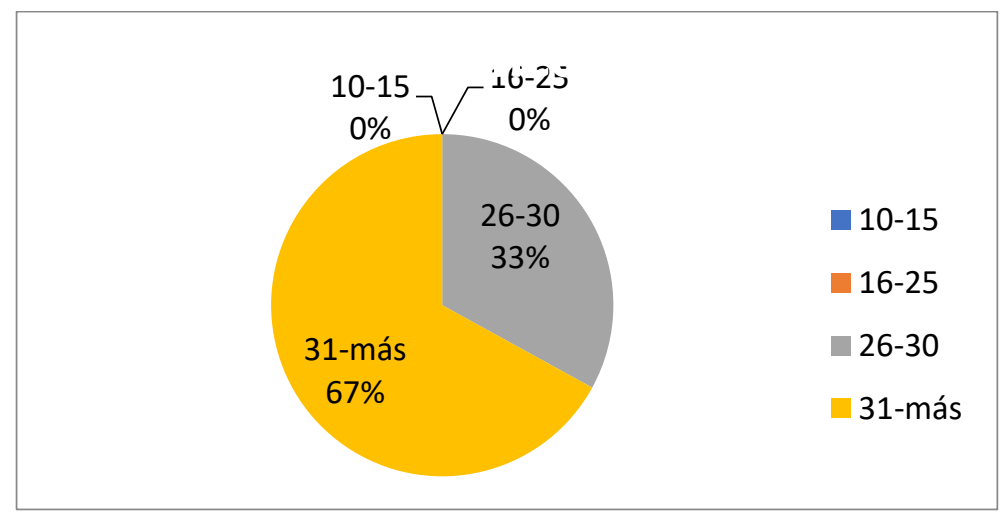

Fuente: Elaboración propia. 
Figura 13. ¿Se sienten cómodos los maestros con la cantidad de estudiantes con los que trabajan?

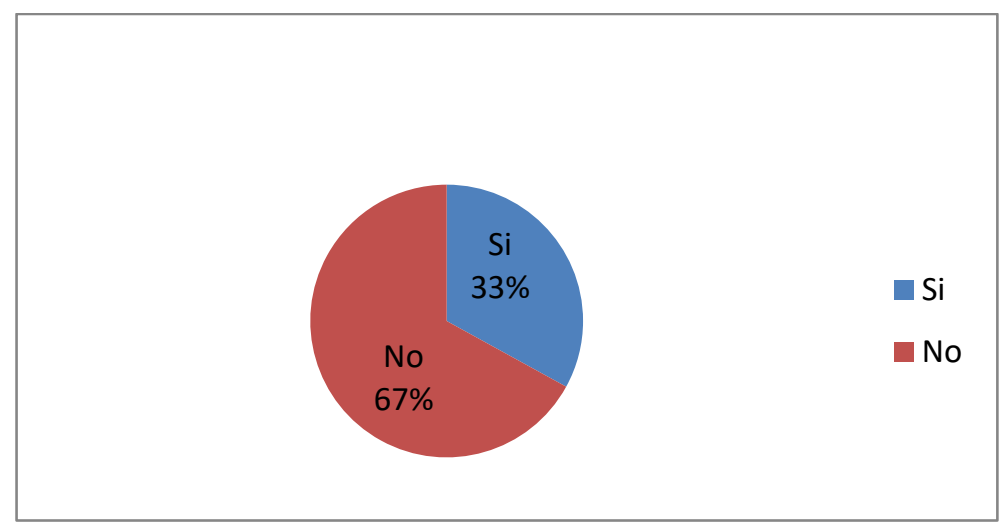

Fuente: Elaboración propia.

Figura 14. ¿Los maestros tienen suficiente espacio para trabajar con el grupo de estudiantes que han sido asignados?

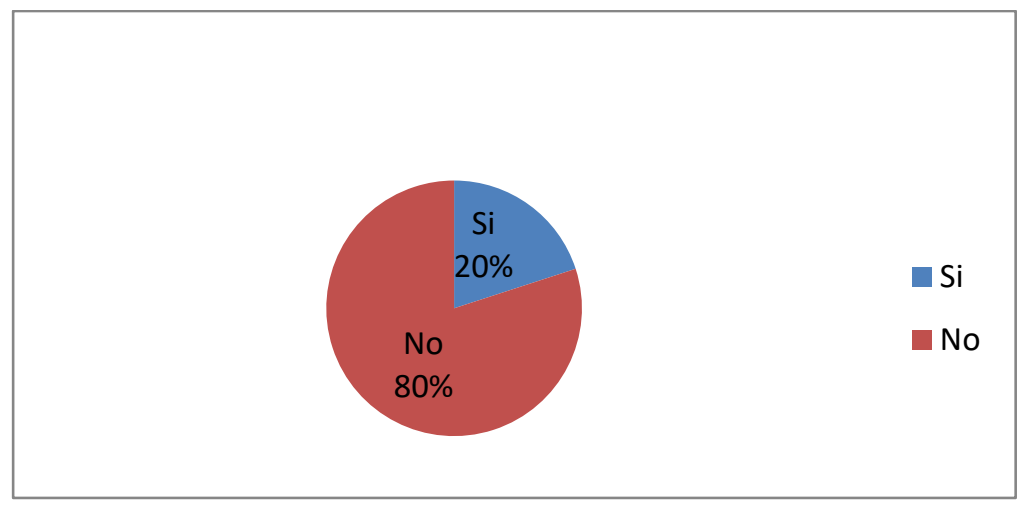

Fuente: Elaboración propia.

Figura 15. ¿Los maestros organizan los asientos de los estudiantes en relación con las actividades planificadas para sus clases?

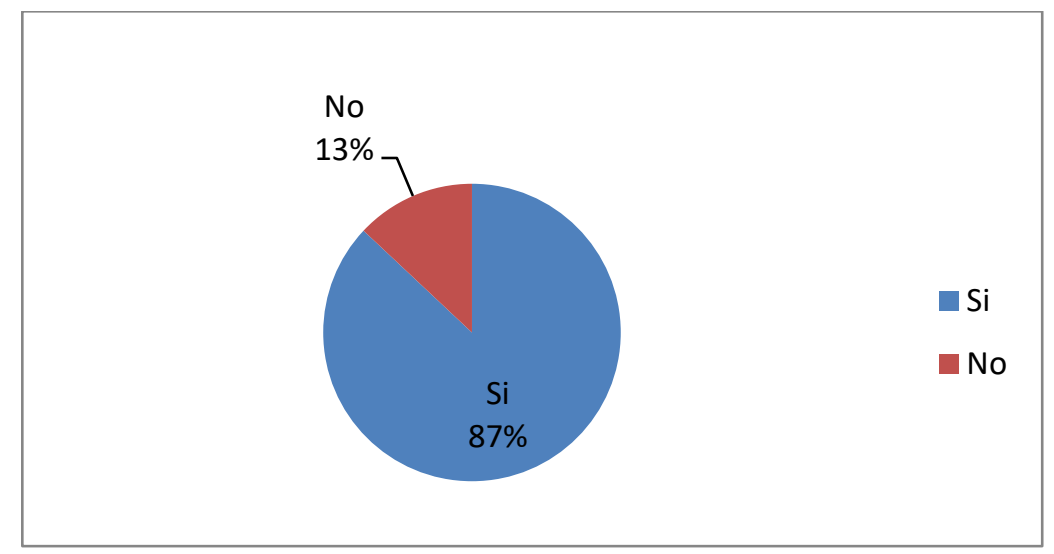

Fuente: Elaboración propia. 
Figura 16. ¿Cuántos alumnos piensan los maestros que es el número apropiado para enseñar inglés?

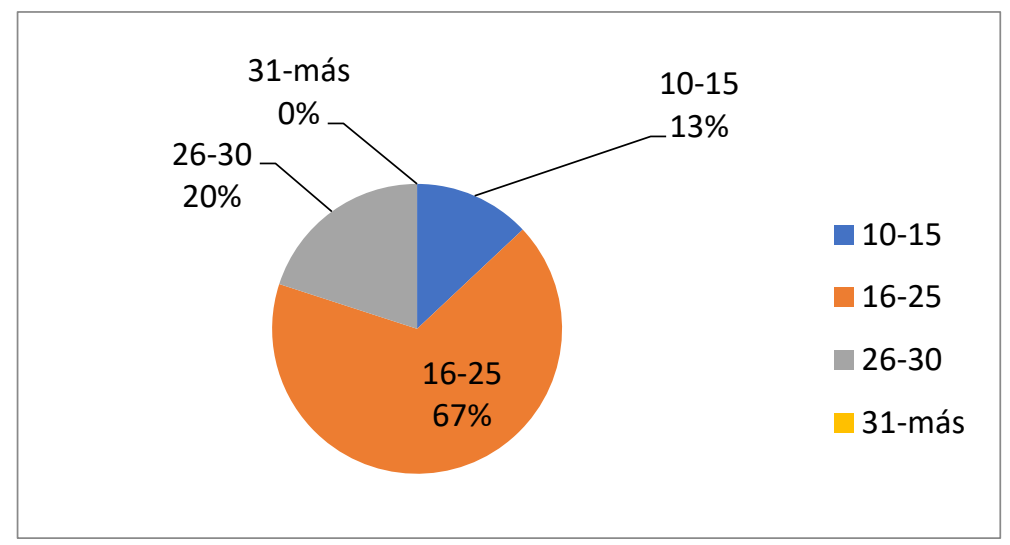

Fuente: Elaboración propia.

Figura 17. ¿Los maestros utilizan recursos de enseñanza (TV, grabadora de cintas / $\mathrm{CD}$, computadora (s), proyector, pizarra inteligente y materiales complementarios?

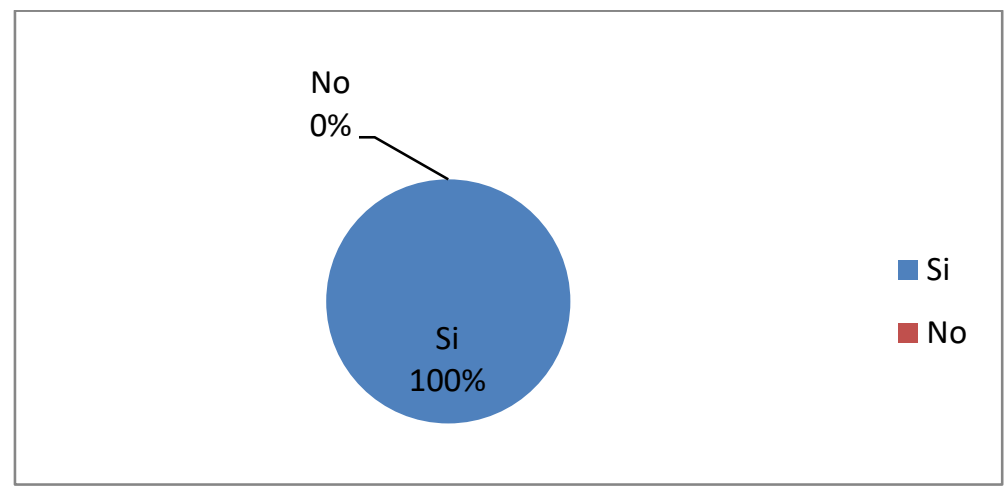

Fuente: Elaboración propia

Figura 18. ¿Los maestros consideran apropiados los recursos que tienen en clase?

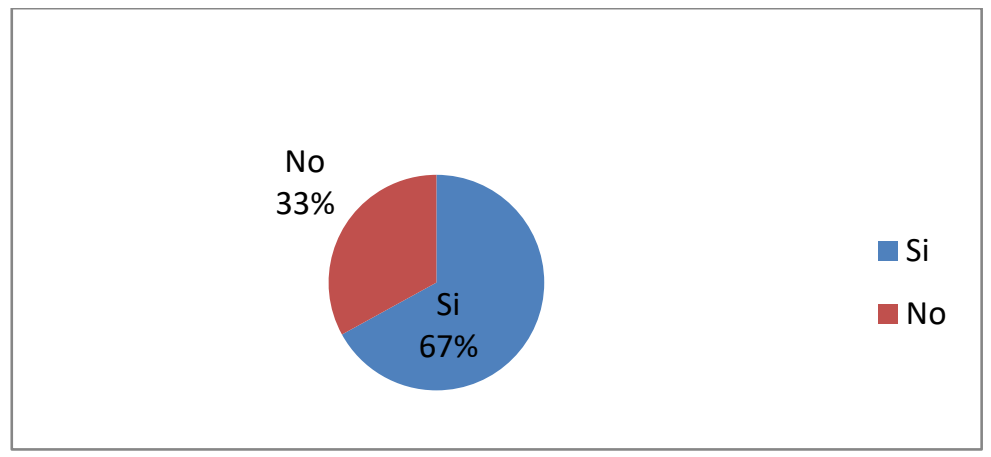

Fuente: Elaboración propia.

Factores concernientes a las instituciones educativas 
Figura 19. ¿Las instituciones revisan los planes de lecciones de los maestros?

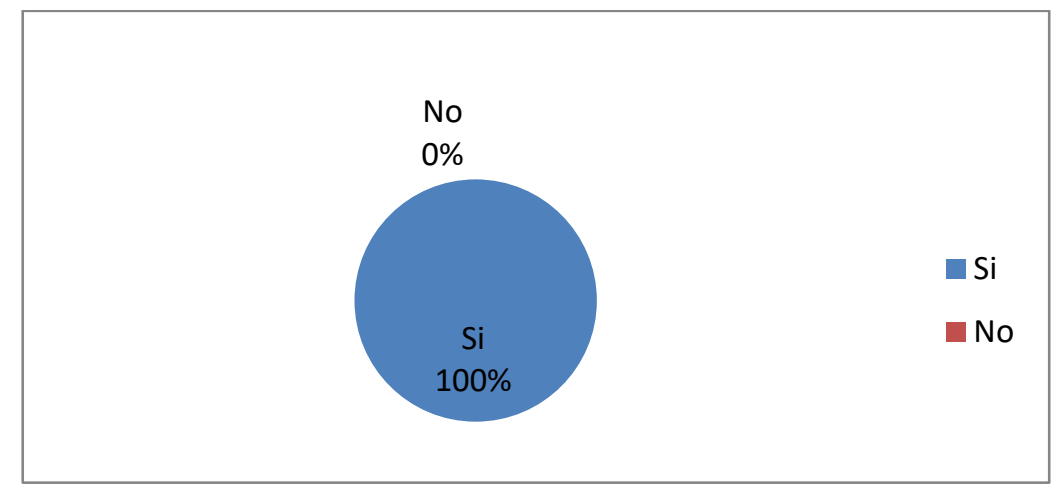

Fuente: Elaboración propia

Figura 20. ¿Las instituciones monitorean las clases de los docentes?

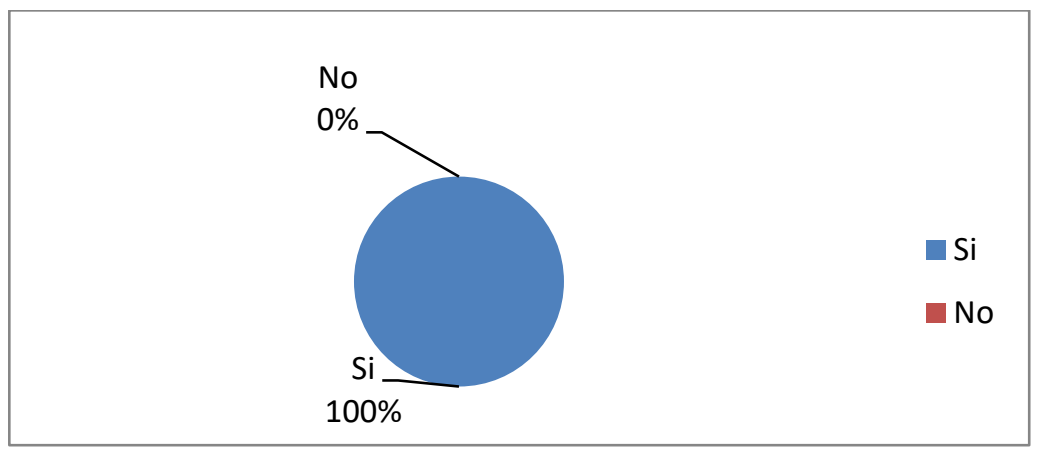

Fuente: Elaboración propia.

\section{Conclusiones:}

- Este estudio evidencia que los dos factores principales que afectan el proceso de enseñanza-aprendizaje de inglés en las escuelas secundarias públicas ecuatorianas son el tamaño de la clase y el número de estudiantes en las aulas.

- Con respecto a los métodos de enseñanza aplicados por los maestros en las aulas, el estudio revela que el más utilizado fue el método de enseñanza de lenguaje comunicativo, que se utilizó para mejorar una habilidad receptiva como la capacidad de escuchar y la habilidad productiva como hablar, que se utiliza en la comunicación real.

- Los datos del estudio muestran que el espacio en el aula en las escuelas secundarias públicas ecuatorianas no permite el desarrollo de una educación adecuada porque no cuentan con el entorno físico apropiado según el número de estudiantes de cada clase.

- Este estudio revela la falta de planificación, que en algunas circunstancias es la ausencia de la preparación de las lecciones diarias por parte de los profesores. 
- Esta investigación evidencia que la mayoría de las escuelas secundarias públicas ecuatorianas no cuentan con los recursos y equipos suficientes para trabajar, como computadoras, pizarras inteligentes, proyectores, materiales lúdicos, etc.

\section{Referencias bibliográficas:}

Abella, D., Salinas, Y. (2006). A case study of the learning styles in low-level learners in a private school in Bogotá. Profile7. 117-129. Retrieved from http://www.scielo.org.co/scielo.php?script=sci_arttext\&pid=S165707902006000100009\&lng=en\&nrm=iso.

Ames, C. \& Archer, J. (1988). Achievement Goals in the Classroom: Student's Learning

Strategies and Motivation Processes. Journal of educational psychology. 80. 3, 260-267. Retrieved from:

http://www.unco.edu/cebs/psychology/kevinpugh/motivation_project/resources/ames_archer 88.pdf

Attardo, S., \& Brown, S. (2005). Understanding language structure, interaction, and variation: An introduction to applied linguistics and sociolinguistics for nonspecialists (2nd ed.). Michigan: The University of Michigan Press.

Blatchford, P. (2003). The class size debate: is small better? Retrieved from http://books.google.com.ec/books/about/The_Class_Size_Debate.html?id=b8_P1thg1 EcC\&redir_esc $=y$

Brookhart, S. M. (2008). How to give effective feedback to your students. Alexandria: Association for Supervision and Curriculum Development

Celce, M. (1991). Teaching English as a second or foreign language: Planning lessons and units (2nd ed.). Boston: Heinle\&Heinle Publishers.

Clark, L. H., \& Starr, I. S. (1981). Secondary and middle school teaching methods (4th ed.). New York: Macmillan Publishing Company Co., Inc.

Cook, V. (1991). Second language learning and language teaching. New York: Edward Arnold Press.

Dörnyei, Z., \& Murphey. T. (2003). Group dynamics in the language classroom. Cambridge: The University of Cambridge Press.

Finn, J. D., \& Achilles, C. M., Mentac R. (1999). Tennessee's class size study: findings, implications, misconceptions. Educational Evaluation and Policy Analysis, 21. 97-109. Retrieved 
http://misd.k12.wa.us/departments/superintendent/class_size/documents/FinnAchilles. pdf

Finocchiaro, M. (1964). English as a second language: From theory to practice. New York: Regents Publishing Company.

Fleta, G.M.T (2006).Aprendizaje y técnicas de enseñanza del inglés. Encuentro, 16, 51-62.

Gower, R., Phillips D., \& Walters, S. (1983). Teaching practice: A handbook for teachers in training. Oxford: Macmillan Publishers Limited

Harmer, J. (2007). The practice of English language teaching (4th ed.). Harlow: Pearson Education Limited.

Harmer, J. (2007). How to teach English. Harlow: Pearson Education Limited.

Heindselman, H., Mentac R. \& Wesler, K. (2007). The effect of classroom seating arrangement learning ability. PSY 220: Research Design and Statistics. 1-12. Retrieved from http://vault.hanover.edu/ altermattw/methods/assets/posterpics/Winter2007/Heindsel man,Mentac,Wesler-w07.pdf

Kauchak, D. P. \& Eggen P. D. (1993). Learning and teaching: Research-based methods. Needham: Allyn and Bacon

Krahnke, K. (1987). Approaches to Syllabus Design for Foreign LanguageTeaching. New York: Prentice Hall.

Lightbown, P. M. \& Spada, N. (1999). How languages are learned (2nd edition). Oxford: Oxford University Press.

Lightbown, P., \& Spada, N. (2006). How languages are learned (3rd ed.). New York: Oxford University Press.

Nunan, D. (1989). Understanding language classrooms: A guide for teacher-initiated action. Hertfordshire: Prentice Hall International.

Olsen, R., \& Kagan, S. (1992). Cooperative Language Learning: A Teacher's Resource Book. New York: Prentice Hall.

Richards, J. C., \& Rodgers, T. S. (2001). Approaches and methods in language teaching. New York: Cambridge University Press

Saville, M. (2006). Introducing a second language acquisition. New York: Cambridge University Press.

Schreiner E. ( $\mathrm{n}$ d). Effective Methods of Teaching Adolescents. Retrieved from: http://www.ehow.com/way_5721466_effective-methods-teaching-adolescents.html 
Scrivener, J. (1994). Learning teaching: A guidebook for English language teachers. Oxford: Macmillan Publishers Limited.

Senescyt (2012). Resolución No. 2012-009: Bases de postulación para el programa de becas "Docentes de Inglés para 8vo. Año de Educación General Básica (EGB) a 3ro. de Bachillerato en Establecimientos Fiscales - Enseña Inglés”. Quito

Snow, S. E. (2002). Teachers' perceptions and use of classroom space. 1-130. Retrieved from: http://www.coe.uga.edu/sdpl/research/teachersperceptions.htm

Wallace, M. J., (1991). Training foreign language teachers: A reflective approach. Cambridge: Cambridge University Press.

Woodward, T. (2001). Planning lessons and courses: Designing sequences of work for the language classroom. New York: Cambridge University Press.

Woolfolk, A. (1980). Educational psychology (10th ed.). Boston: Pearson Education. 


\section{PARA CITAR EL ARTÍCULO INDEXADO.}

Páez Romero, V. G., \& Santillán Marroquín, W. E. (2020). Factores que afectan el aprendizaje del idioma inglés en colegios públicos de Quito. Explorador Digital, 4(3), 32-46. https://doi.org/10.33262/exploradordigital.v4i3.1278

\section{Ciencia \\ LDigital}

El artículo que se publica es de exclusiva responsabilidad de los autores y no necesariamente reflejan el pensamiento de la Revista Explorador Digital.

El artículo queda en propiedad de la revista y, por tanto, su publicación parcial y/o total en otro medio tiene que ser autorizado por el director de la Revista Explorador Digital.
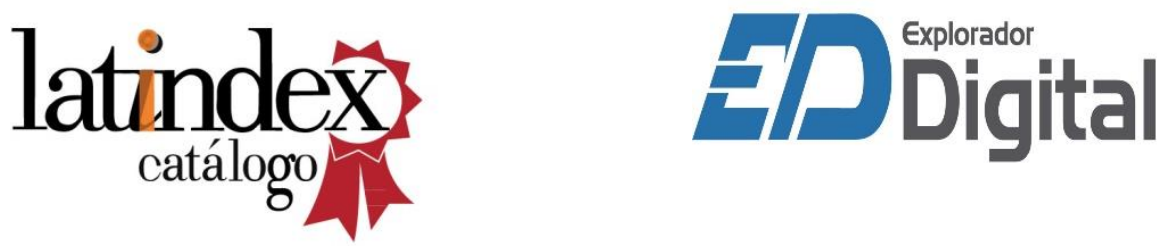